Boise State University

ScholarWorks

Civil Engineering Faculty Publications and

Presentations

Department of Civil Engineering

$12-1-2012$

Experimental Validation of a Numerical Forward Model for Tunnel Detection Using Cross-Borehole Radar

Arvin Farid

Boise State University

Jose A. Martinez-Lorenzo

Northeastern University

Akram N. Alshawabkeh

Northeastern University

Carey M. Rappaport

Northeastern University 


\title{
EXPERIMENTAL VALIDATION OF A NUMERICAL FORWARD MODEL FOR TUNNEL DETECTION USING CROSS-BOREHOLE RADAR
}

\section{Arvin Farid ${ }^{1}$, M.ASCE, Jose A. Martinez-Lorenzo ${ }^{2}$, Akram N. Alshawabkeh ${ }^{3}$, M.ASCE, and Carey M. Rappaport ${ }^{4}$}

\begin{abstract}
The goal of this research is to develop an experimentally validated twodimensional (2D) finite difference frequency domain (FDFD) numerical forward model to study the potential of radar-based tunnel detection. Tunnel detection has become a subject of interest to the nation due to the use of tunnels by illegal immigrants, smugglers, prisoners, assailants, and terrorists. These concerns call for research to nondestructively detect, localize, and monitor tunnels. Nondestructive detection requires robust image reconstruction and inverse models, which in turn need robust forward models. Cross-Well Radar (CWR) modality is used for experimentation to avoid soil-air interface roughness. CWR is not a versatile field technology for political boundaries but is still applicable to monitoring the perimeter of buildings or secure sites. Multiple-depth wideband frequency-response measurements are experimentally collected in fully water-saturated sand, across PVC-cased ferrite-bead-jacketed borehole monopole antennae at a pilot scale facility (referred to as SoilBED). The experimental results are then compared with the 2D-FDFD model. The agreement between the results of the numerical and experimental simulations is then evaluated. Results of this work provide key diagnostic tools that can help to

${ }^{1}$ Assistant Professor, Civil Engineering, Boise University, Boise, ID 83725-2060, Phone: (208) 426-4827, Fax: (208) 426-2351- Email: ArvinFarid@BoiseState.edu

2 Research Assistant Professor, Electrical \& Computer Eng., Gordon CenSSIS, Northeastern University, Boston, MA 02115, Email: jmartine@ece.neu.edu

${ }^{3}$ Professor, Civil \& Environmental Eng., Northeastern University, Boston, MA 02115, Email: aalsha@coe.neu.edu

${ }^{4}$ Professor, Electrical \& Computer Eng., Gordon CenSSIS, Northeastern University, Boston, MA 02115, Email: rappaport@ece.neu.edu
\end{abstract}


develop the algorithms needed for the detection of underground tunnels using radar-based methods.

CE Database subject headings: Tunnel Detection; Radar; Forward Model; CWR; GPR

\section{INTRODUCTION}

Tunnels present both military and homeland security threats. The discovery of these tunnels has historically relied on old-fashioned techniques of using informants, tips, and surveillance. Many nondestructive approaches for tunnel detection (e.g., seismic-acoustic methods, resistivity, ground-penetrating radar, microgravity, magnetic methods) have been proposed and researched. However, the application of a practical, reliable, and easily deployable method remains a significant challenge (Army Research Office 2006) of importance to the Department of Homeland Security.

Mahrer and List (1995) used three electromagnetic procedures to detect and localize a $74 \mathrm{~cm}$ $\times 150 \mathrm{~cm}$ tunnel at a depth of $1400 \mathrm{~cm}$ across the U.S.-Mexico border near Otay Mesa, east of San Diego, CA, and achieved an acceptable level of success. They used: (i) a surface-to-surface study over $22 \mathrm{kHz}$ to $300 \mathrm{kHz}$; (ii) a surface-to-borehole study at approximately $100 \mathrm{kHz}$; and (iii) a borehole-to-borehole study with zero-offset (i.e., transmitter and receiver at the same depth) traverses between $1 \mathrm{MHz}$ to $15 \mathrm{MHz}$.

Cross-borehole radar (in the $\mathrm{MHz}$ to $\mathrm{GHz}$ range) was also proven efficient when it confirmed the existence of a deep tunnel through crystalline rock in the southern part of the Demilitarized Zone in the Republic of Korea (Allen 1992). Different theoretical models capable of simulating EM-wave propagation exist, some commercially available. For example, Bissessur and Peyton (2001) used a commercially available parameterized finite element model for image 
reconstruction through electromagnetic inductance tomography. Such commercial models are general models, not scientifically flexible to model the geometry or dispersive (frequencydependent) nature of lossy soils. A lossy medium is a medium in which EM energy is absorbed or dissipated. Another problem is the computational time and cost for real-time image reconstruction. Detection and localization of tunnels also require inversion and image reconstruction. Solving the inverse problem requires a robust forward model to simulate EMwave propagation and scattering by the tunnel. Inversion and tomographic-image reconstruction do not fit within the scope of this note. A 2D finite difference frequency domain (2D-FDFD) model is used in this work to model cross-well radar (CWR), which is then experimentally validated. CWR uses antennas lowered into boreholes while GPR works above ground. Therefore, in this laboratory work, the CWR method has been selected to study the potential of using radar-based methods such as GPR — for along political boundaries - and cross-well radar (CWR) — for around buildings - as well as reducing the noise due to the roughness of the soilair interface in the case of GPR. The numerical model is developed and validated through comparison with an experimentally simulated scattered field due to tunnels within a fully watersaturated, poorly graded sand medium shown in Fig. 1.

\section{FDFD SIMULATION}

Due to the large length of tunnels relative to their diameter, the problem is assumed to be a twodimensional transverse-electric (TE) mode. Transverse electric (TE) means only the X- and Ycomponents $\left(E_{x}\right.$ and $\left.E_{y}\right)$ of the electric (E) field in the cross-sectional plane of the tunnel and the $H_{z}$ component of the magnetic $(\mathrm{H})$ field perpendicular to this plane exist. In this case, the $\mathrm{X}$-axis is horizontal and across the tunnel, the Z-axis is horizontal and parallel to the tunnel 
(perpendicular to the paper), and the Y-axis is vertical. This 2D TE-mode problem is simulated using a 2D-FDFD technique. EM-wave propagation is governed by Maxwell's equations (Grant and Philips 1990; Sheriff 1989; and Bergmann et al. 1998). Assuming solutions to the governing equations with harmonic time dependence $\left(e^{-j \omega t}\right)$, the Maxwell's curl equations for electric and magnetic fields can be merged into Helmholtz's equation of the following form,

$$
\left(\nabla^{2}+k^{2}\right) H_{z}=\frac{1}{k^{2}} \frac{\partial H_{z}}{\partial y} \frac{\partial\left(k^{2}\right)}{\partial y}+\frac{1}{k^{2}} \frac{\partial H_{z}}{\partial x} \frac{\partial\left(k^{2}\right)}{\partial x}
$$

where $H_{z}=$ Z-component of the magnetic field, $k=$ wave number $=\sqrt{\omega \varepsilon_{c} \mu}$, where, in turn, $\varepsilon_{c}=$ complex dielectric permittivity $=\varepsilon^{\prime}-j \sigma_{e} / \omega, \varepsilon^{\prime}=$ real dielectric permittivity, $\omega=$ angular frequency, $\mu=$ magnetic permeability, $\sigma_{e}=$ effective electrical conductivity, and $j=\sqrt{-1}$. Equation (1) can be spatially discretized to the following form using a central finite difference formulation (Rappaport et al. 2004),

$$
\begin{aligned}
& \frac{1}{\Delta^{2}}\left\{\left(H_{z, i+1, j}-2 H_{z, i, j}+H_{z, i-1, j}\right)+\left(H_{z, i, j+1}-2 H_{z, i, j}+H_{z, i, j-1}\right)\right\}+k_{i, j}^{2} H_{z, i, j}= \\
& \frac{1}{4 \Delta^{2} k_{i, j}^{2}}\left\{\left(k_{i+1, j}^{2}-k_{i-1, j}^{2}\right)\left(H_{z, i+1, j}-H_{z, i-1, j}\right)+\left(k_{i, j+1}^{2}-k_{i, j-1}^{2}\right)\left(H_{z, i, j+1}-H_{z, i, j-1}\right)\right\}
\end{aligned}
$$

where a $170 \times 200$ rectangular uniform grid (with a grid size of $\Delta x=\Delta y=\Delta=0.2 \mathrm{~cm}$ ) is used. Subscripts $i$ and $j$ represent the node number on the medium discretization grid in $\mathrm{X}$ and $\mathrm{Y}$ directions. As seen in Fig. 1, the source was modeled as two 5.1-cm-long uniform arrays of unitmagnitude $\left(\left|H_{z}\right|=1 \mathrm{Amp} / \mathrm{m}\right.$, ) magnetic dipoles, located perpendicular to the figure page, vertically across the position of the transmitter antenna, three grid-sizes $(0.6 \mathrm{~cm})$ apart (i.e., two legs of each dipole array are $0.6 \mathrm{~cm}$ apart in the Z-direction, and the two dipole arrays are $0.6 \mathrm{~cm}$ apart in the Y-direction). The two vectors are at a $180^{\circ}$ phase shift (i.e., opposite sign / direction) with respect to each other (i.e., the other 5.1-cm-long uniform dipole array). The actual dielectric properties of the saturated sand used in the FDFD simulation come from another work by the 
authors (Zhan et al. 2007). In that work, published values (Von Hippel 1954; and Hipp 1974) for dielectric properties were selected as the first guess, then optimized to match results of another analytical simulation (a half-space lossy Green's function, Zhan et al. 2007) to the experimental measurements collected using the setup of Fig. 1. The real relative dielectric permittivity $\left(\varepsilon_{r}{ }_{r}=\right.$ $\varepsilon^{\prime} / \varepsilon_{0}$, where $\varepsilon_{0}$ is the real dielectric permittivity of free-space) and loss tangent $(\tan \delta)$ of the saturated sand at $1.093 \mathrm{GHz}$ used in the simulation are 17.5 and 0.12 , respectively. A sensitivity analysis (to evaluate the sensitivity of the simulation results to the input dielectric properties) was performed, which does not fit within the scope of this technical note. Equation (2) is solved for the magnetic field $\left(H_{z}\right)$ at all grid points. Then, using Equations (3) and (4) (extracted from Ampere's law), the electric field ( $E_{x}$ and $\left.E_{y}\right)$ can be computed based on $\mathrm{H}_{\mathrm{z}}$ at all grid-points:

$$
\begin{aligned}
& E_{x}=\frac{1}{j \omega \varepsilon_{c}} \frac{\partial H_{z}}{\partial \mathrm{y}} \\
& E_{y}=\frac{-1}{j \omega \varepsilon_{c}} \frac{\partial H_{z}}{\partial \mathrm{x}}
\end{aligned}
$$

\section{EXPERIMENTAL SETUP}

The SoilBED facility of The Gordon Center for Subsurface Sensing and Imaging Center houses a 121.9-cm-deep, 152.4-cm-wide, and 274.3-cm-long $(4 \mathrm{ft} \times 5 \mathrm{ft} \times 9 \mathrm{ft})$ soil box with a controlled drainage system (Fig. 1). The soil in the facility was fully water-saturated, poorly graded sand (SP) and loosely packed (for more information about the grain-size distribution as well as physical and dielectric properties of the sand, refer to Farid et al. 2006). The soil was saturated from the bottom, and the water table was maintained at the soil-air interface to reach as close to full water-saturation as possible. The drainage system consists of a network of perforated PVC pipes wrapped in geotextile fabric filter buried underneath a thin gravel layer (for more information about the experimentation, its challenges, and solutions, refer to Farid et al. 2006). A 
137.2-cm-long PVC pipe with an outer diameter of $3.8 \mathrm{~cm}$ and wall thickness of $0.16 \mathrm{~cm}$ was water-sealed (i.e., watertight, and no leakage of water is allowed into or out of the pipe) at its ends and used to simulate an air-filled tunnel. PVC was selected due to its thin PVC wall with a similar relative dielectric permittivity to the one of air $\left(\varepsilon_{r, P V C}^{\prime} \approx 2.55\right.$ and $\left.\varepsilon_{r, a i r}^{\prime}=1\right)$. The PVCcased tunnel was then horizontally buried within the SoilBED at a depth of $23 \mathrm{~cm}$ (from the center of the pipe). As seen in Fig. 1, two PVC-cased ferrite-bead-jacketed borehole monopole antennae were installed vertically across the tunnel, in a way that the center of the tunnel was 20 $\mathrm{cm}$ and $10 \mathrm{~cm}$ apart from the transmitting and receiving antennae, respectively. The diameter of the PVC casings for antennae was $1.9 \mathrm{~cm}$, and its thickness was $0.16 \mathrm{~cm}$. The transmitting and receiving monopole antennae were each 0.35 -cm-thick rigid copper coaxial (RG 402) cables with $5.1 \mathrm{~cm}$ of their shield stripped at the end to have a resonant frequency of $1.093 \mathrm{GHz}$ (for more information, refer to Farid et al. 2006). As seen in Fig. 1, another identical transmitting antenna was installed $30 \mathrm{~cm}$ from the receiver, in a way that the medium between the receiver and this identical transmitter was entirely filled with homogeneous soil to simulate a replica of the experiment background without any tunnel object, sharing the same receiver.

To minimize heterogeneity and create a homogeneous background, the sand within $45 \mathrm{~cm}$ (in all directions) from the center of the tunnel was excavated (Fig. 1) and then uniformly remixed after the placement of the tunnel and antennae. After installing the PVC-cased tunnel and the casing for the three monopole-antennae, the sand was carefully leveled. The sand was saturated and drained several times to slightly compact the soil and eliminate the possibility of future compaction after repeated drainage and saturation before each experiment. This plan was selected merely due to the result of the authors' observation that repeated drainage and saturation in advance of experimentation can uniformly densify the soil and eliminate unwanted localized 
compaction during packing of the soil. This helps to acquire repeatable frequency-response measurements. Fig. 1 illustrates a schematic and a picture of the experiment. A Vector Network Analyzer (Agilent 8714ES) and a 12-channel multiport test-set (Agilent 8750) were used to collect multiple-depth transmission and reflection S-measurements $\left(S_{11}=\right.$ reflection frequencyresponse at Antenna 1, the transceiving (i.e., both transmitting and receiving) port/antenna; and $S_{21}=$ transmission frequency-response from Antenna 1, the transmitting port/antenna, to Antenna 2, the receiving port/antenna; for more information, refer to Farid et al. 2006). The transmitters' depths were varied from $19 \mathrm{~cm}$ to $31 \mathrm{~cm}$, at $6 \mathrm{~cm}$ intervals. The receiver depth was varied between $11 \mathrm{~cm}$ and $35 \mathrm{~cm}$, at $2 \mathrm{~cm}$ intervals. Sample ray-paths for some of these depthcombinations of antennae are shown in Fig. 1. The scattered field due to the tunnel was computed by subtracting the background (incident) field (i.e., the electric field through the soil without the tunnel anomaly) from the total field (i.e., the measurement for the soil with the tunnel). The fact that the tunnel visibly scatters (e.g., reflects, refracts, and/or diffracts) the field is not sufficient for the image reconstruction. The next step is to validate this forward model against experimentation.

\section{VALIDATION OF FORWARD MODEL VIA EXPERIMENTATION}

Both the transmitting and one receiving antennae are vertically polarized monopole antennae, which have much stronger reception in the vertical (Y) direction. Therefore, the Y-component of the electric field $\left(E_{y}\right)$ is stronger and, hence, selected to be shown in all illustrations. On the other hand, the experimental results are measured at only 2-cm receiver depth intervals; while the simulation shows the results at every grid point $(0.2 \mathrm{~cm}$ rectangular grid $)$ and can be visualized continuously in diagrams. Fig. 2 shows the simulated Y-component of the incident (soil without 
tunnel) electric field radiated from the transmitting antenna (located at $X=-20 \mathrm{~cm}, Y=-19 \mathrm{~cm}$ ) on a vertical (depth) slice. Fig. 3, on the other hand, shows the simulated Y-component of the scattered electric field $\left(E_{y}\right)$ due to the tunnel for the three transmitter depths of $19 \mathrm{~cm}, 25 \mathrm{~cm}$, and $31 \mathrm{~cm}$ on a vertical (depth) slice. The tunnel acts as a secondary source of the scattered field, visible in the simulated results in Fig. 3. The location and depth of the source of incident field control the radiation pattern of the scattered field.

The simulated results need to be compared with the experimental ones to assess the degree of agreement. Subtracting the incident field from the total field will result in the scattered field. This can be performed for the three cases with transmitter depths of $19 \mathrm{~cm}, 25 \mathrm{~cm}$, and $31 \mathrm{~cm}$ (Fig. 4). As seen in Fig. 4, for the transmitter at these three depths, the scattering effect of the tunnel on EM-wave propagation becomes strongly visible when the receivers are at depths closer to the tunnel depth. The peaks of the experimental and simulated scattered field magnitudes also coincide with the depth of the tunnel visible in the radiation pattern simulation in Figs. 3 and 4 for the three transmitter depths of these three figures. The pattern of simulated and experimental scattered fields for the source of incident field (i.e., transmitting antennae) at all three depths show a visible similarity; however, there are some discrepancies between the peak values of the experimental and simulated results. These discrepancies may be due to the anisotropic propagation or frequency dispersion associated with sand/saturation variations not accounted for within the numerical model versus the actual variations in the experiment. Even though efforts were focused on creating a well-controlled homogenous soil medium, this cannot be achieved at the level assigned to the numerical model. The disagreement between the experimental and simulated scattered fields of Fig. 4 near the top and bottom can be explained by the strong reflection at the top (air-soil interface) and bottom (soil-concrete interface) of the SoilBED in the 
experimentation. The bottom soil-concrete interface is not modeled in the simulation. The simulation models the soil as an infinite medium using a perfectly matched layer method for boundary conditions and truncation of the discretization grid. It can be deduced from the simulation plots of Figs. 2 through 4 that both the soil-air interface and tunnel caused scatterings of the same order of magnitude on the electric field that couple to each other. When the tunnel is deeper, the surface effect diminishes due to the attenuation of the waves along the longer travel path reflected by the soil-air interface.

\section{CONCLUSIONS}

A 2D-FDFD numerical forward model was developed to simulate radar-based tunnel detection using the CWR method. The numerical model was then validated against a pilot-scale experiment. The CWR method is proven to be a promising solution for tunnel detection and long-term monitoring of perimeters of secured areas against intruders. However, CWR is not versatile enough for broad investigations of a subsurface for anomalies. The reason CWR was selected for this comparison and validation process was to avoid the soil-air interface roughness to create a controlled laboratory medium. Regardless of the depth of the incident field source (i.e. transmitter), the magnitude of the scattered field peaks at the depth of the tunnel. This is a strong representative of the tunnel depth. Another outcome of this study is the close agreement between the experimental and simulated scattered fields. Comparison of the results of this model with other models is necessary and ongoing. While the technique was successful in detecting the

presence and depth of the "experimentally simulated tunnel" in a homogenous ground space, the overall approach needs to be validated progressively for scalability and performance sensitivity to geological heterogeneity. As mentioned, when scaling from the experiment to the field, the 
signal attenuation through soil may not scale linearly, which can affect the feasibility of the

results in the field. Field-scale experimentation is necessary to evaluate this concern. The authors acknowledge that due to discrete limitations and environmental sensitivities of different individual sensing modalities like the one (CWR) presented in this note, sensor fusion (i.e., simultaneous use of various sensors such as radar, acoustics, resistivity) at various modalities (e.g., surface-reflection, cross-borehole, down-hole) may be a more robust approach.

\section{REFERENCES}

Allen, J. W. (1992). "Integrated geophysical approach to the detection of buried objects and clandestine tunnels." The International Society for Optical Engineering, Proceedings of SPIE (Society of Photo-Optical Instrumentation Engineers), 1693, 161-169, August.

Army Research Office, CERDEC-NVESD Countermine Directorate, and RDECOM CIED/CM IPT (2006). Army advanced concept workshop on shallow tunnel detection. Jamie Whitten Nation Center for Physical Acoustics, University of Mississippi, February 14-17.

Bergmann, T., Robertson, J. O. A., and Holliger, K. (1998). "Finite-difference modeling of electromagnetics propagation in dispersive and attenuating media." Geophysics, 63(3), 856867, May-June.

Bissessur, Y, and Peyton, A. J. (2001). "Image reconstruction for electromagnetic inductance tomography employing a parameterized finite-element-based forward model." International Society of Optical Engineering, Proceedings of SPIE (Society of Photo-Optical Instrumentation Engineers), 4188, 261-272 
Farid, A. M., Alshawabkeh, A. N., and Rappaport, C. M. (2006). "Calibration and validation of a laboratory experimental setup for CWR in sand." ASTM (American Society for Testing and Materials), Geotechnical Testing Journal, 29(2), 158-167.

Grant, I. S. and Philips, W. R. (1990). Electromagnetism. John Wiley \& Sons, New York.

Hipp, J. E. (1974). "Soil electromagnetic parameters as functions of frequency, soil density, and soil moisture." Proceedings of the IEEE (Institute of Electrical and Electronics Engineers), 62(1), 98-103.

Mahrer, K. D. and List, D. F. (1995). "Radio frequency electromagnetic tunnel detection and delineation at the Otay Mesa site.” Geophysics, 60(2), 413-422.

Rappaport, C. M., Dong, Q., Bishop, E., Morgenthaler, A., and Kilmer, M. (2004). "Finite Difference Frequency Domain (FDFD) modeling of two-dimensional TE wave propagation and scattering." URSI (Union Radio Scientifique Internationale), International Symposium on Electromagnetics Theory, Pisa, Italy, May 16-18, 1134-1136.

Sheriff, R. E. (1989). Geophysical methods. Prentice Hall, New Jersey.

Von Hippel, A. R. (1954). Dielectric materials and applications. MIT (Massachusetts Institute of Technology) Press, Cambridge, MA.

Zhan, S. H., Farid, A., Alshawabkeh, A. N., Raemer, H., and Rappaport, C. M. (2007). "Validated half-space Green's function formulation for Born approximation for cross-well radar sensing of contaminants." IEEE (Institute of Electrical and Electronics Engineers), Transaction of Geoscience and Remote Sensing, 45(8), 2423-2428, August. 
Fig. 1. Buried tunnel and PVC-cased monopole antennae, photograph and schematic of the cross-section of the experiment

Fig. 2. Magnitude (left) and phase (right) of the Y-component of the simulated incident (soil without tunnel) electric field radiated from the transmitter (at: $X=20 \mathrm{~cm}, Y=-19 \mathrm{~cm}$ )

Fig. 3. Magnitude (left) and phase (right) of the Y-component of the simulated scattered (= total - incident) electric field by the tunnel (located at $X=0 \mathrm{~cm}, Y=-23 \mathrm{~cm}$ ) acting as a secondary source of radiation with a source of incident field (transmitting antenna) at: (a) $X=20 \mathrm{~cm}, Y=$ $19 \mathrm{~cm}$, (b) $X=20 \mathrm{~cm}, Y=-25 \mathrm{~cm}$, and (c) $X=20 \mathrm{~cm}, Y=-31 \mathrm{~cm}$

Fig. 4. Comparison between simulated and experimentally measured Y-component of the scattered (= total - incident) electric fields due to the tunnel (at $X=0 \mathrm{~cm}$ and $Y=-23 \mathrm{~cm}$ ) for the transmitter at: (a) $X=20 \mathrm{~cm}, Y=-19 \mathrm{~cm}$, (b) $X=20 \mathrm{~cm}, Y=25 \mathrm{~cm}$, and (c) $X=20 \mathrm{~cm}, Y=-31$ $\mathrm{cm}$ 


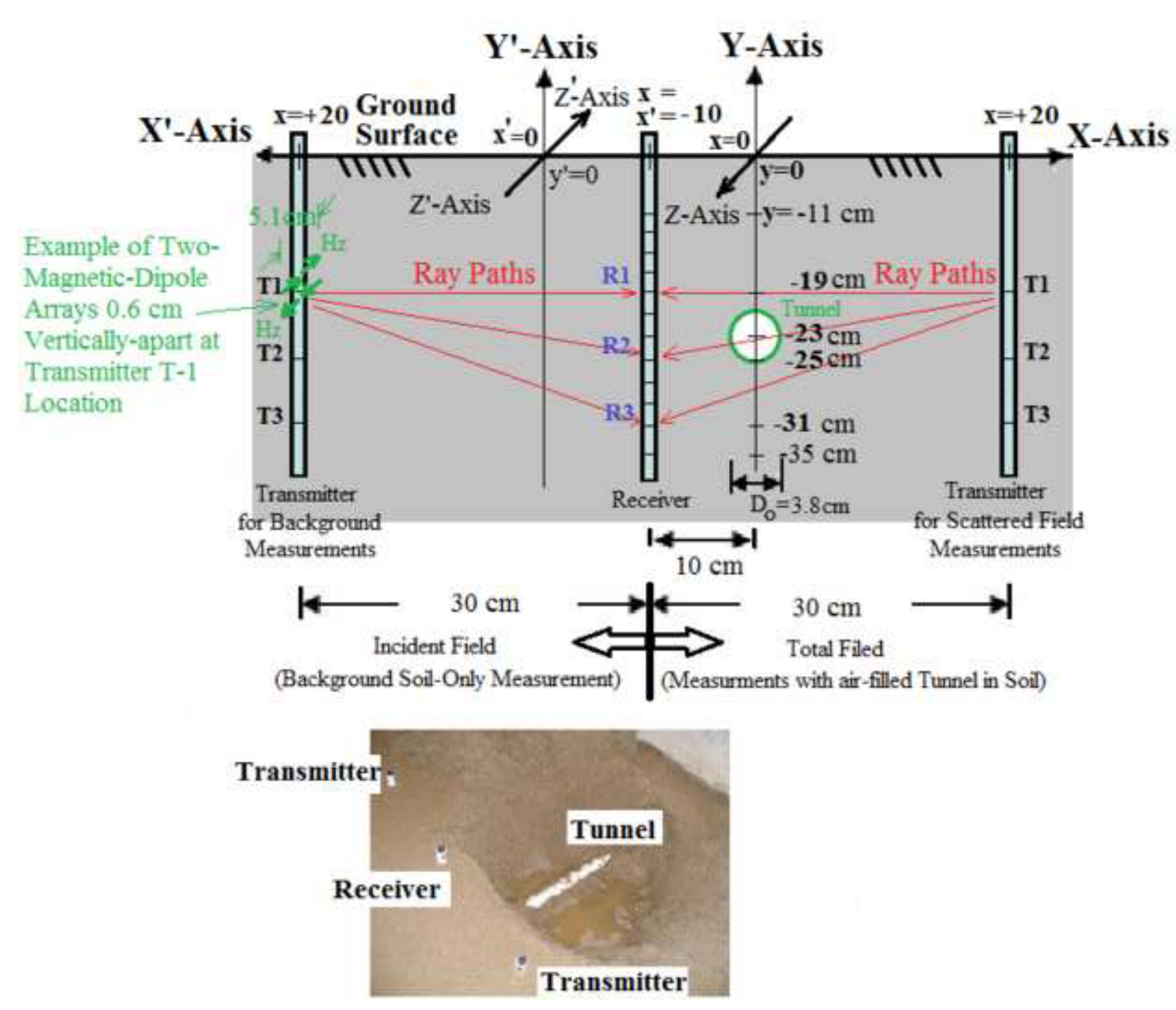



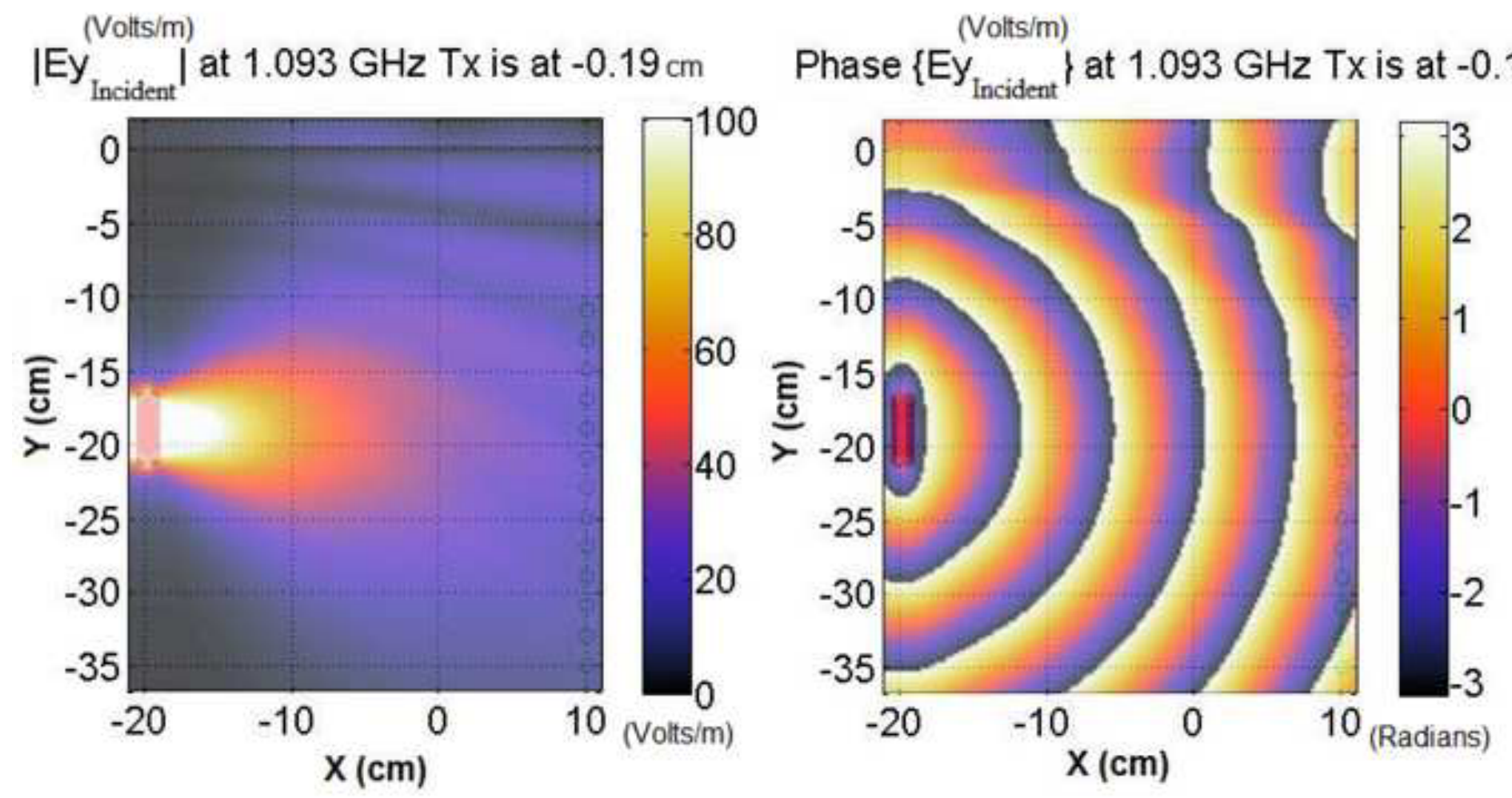
(Volts/m) (Voltsim) $\mid \mathrm{Ey}_{\text {Total }}$ - $\mathrm{Ey}_{\text {Incident }}$ at $1.093 \mathrm{GHz} \mathrm{Tx}$ is at $-0.19 \mathrm{~cm}$ Phase $\left\{\mathrm{Ey}_{\text {Total }}-\mathrm{Ey}_{\text {Incident }}\right\}$ at $1.093 \mathrm{GHz} \mathrm{Tx}$ is at $-0.19 \mathrm{~cm}$
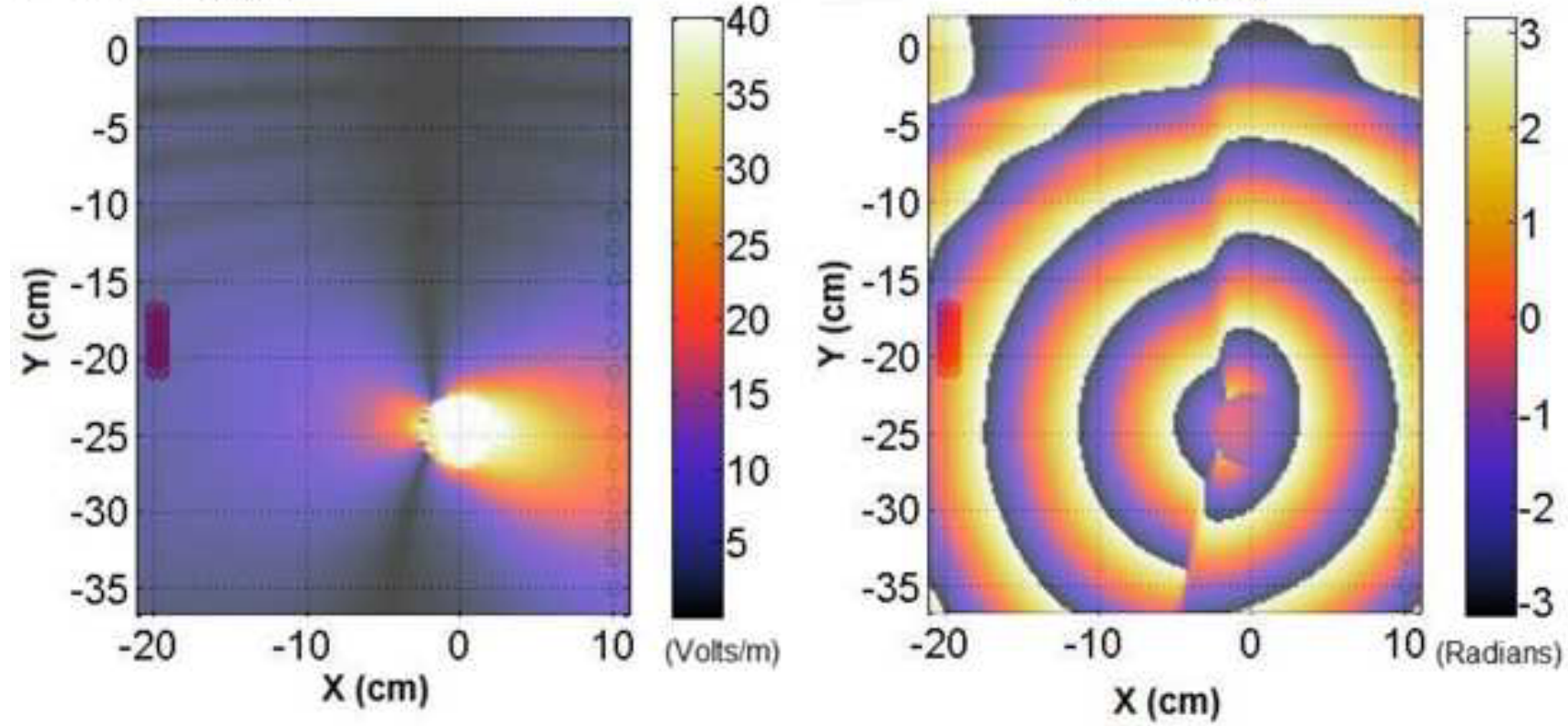
(Volts/m) (Volts/m)

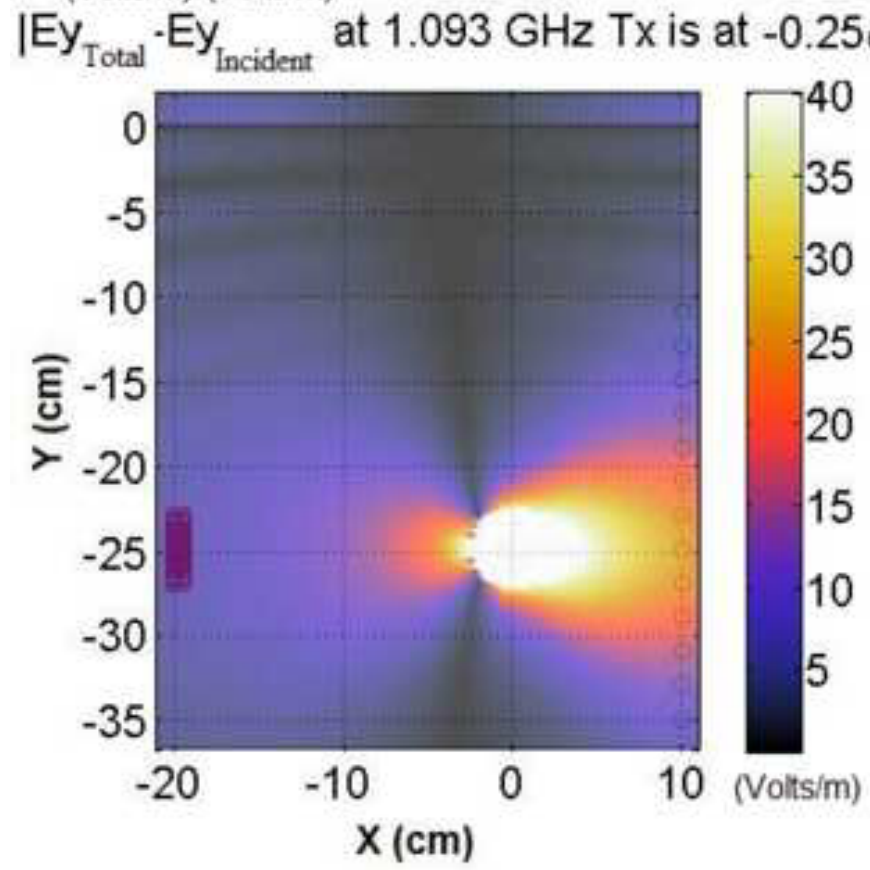

(Volts/m) (Volts/m)
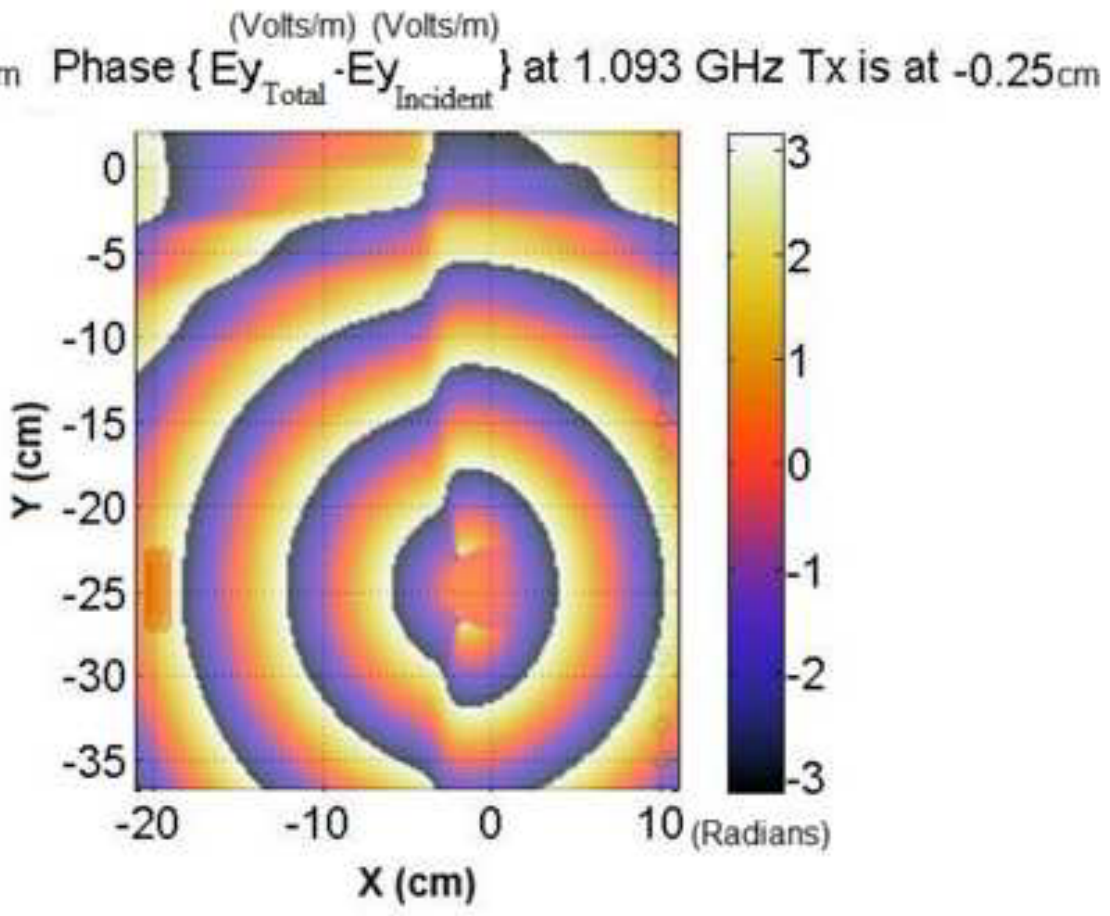

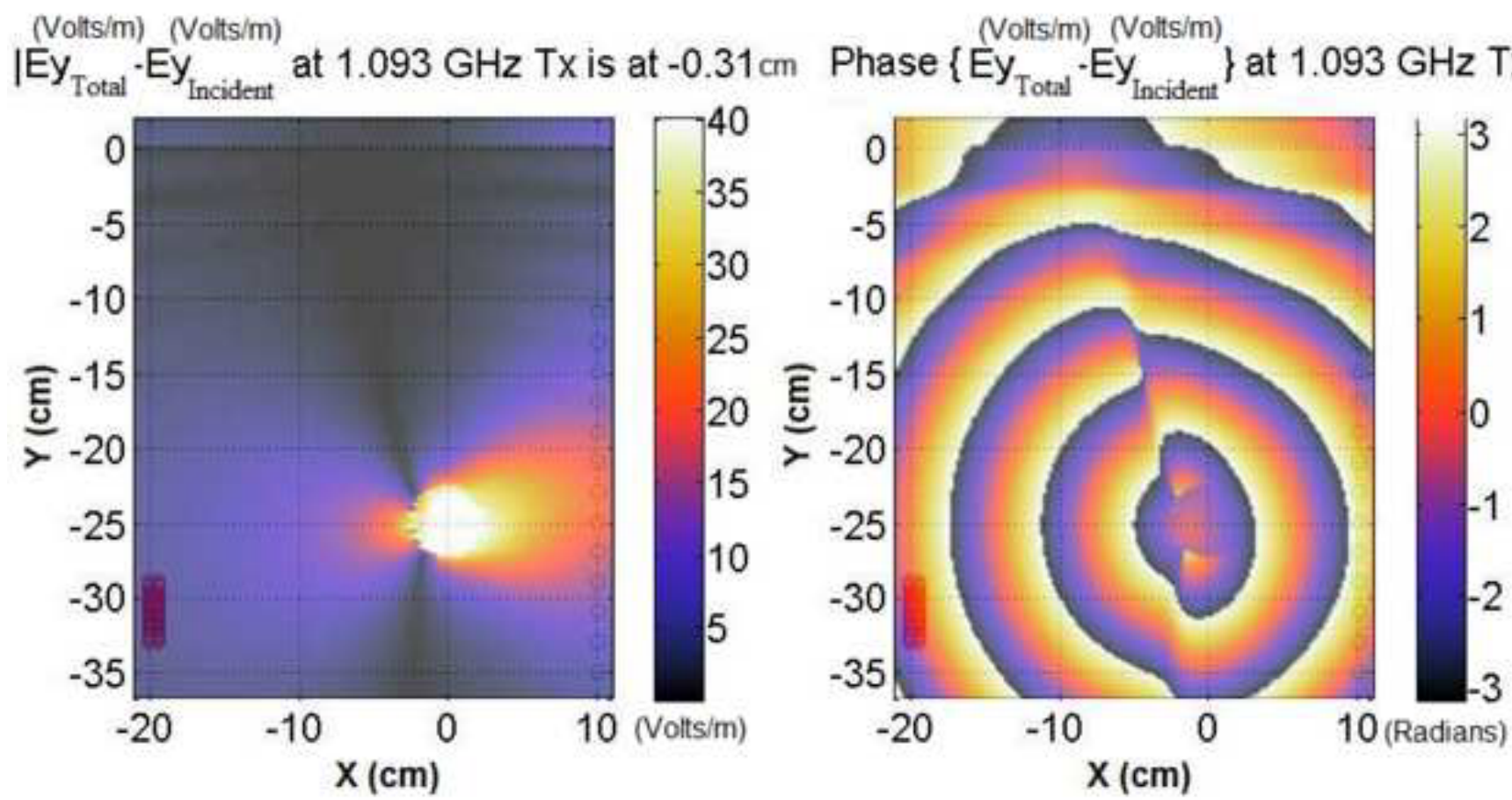


\section{MEASURED vs FDFD at $1.093 \mathrm{GHz}$, Tx is at $-0.19 \mathrm{~cm}$}

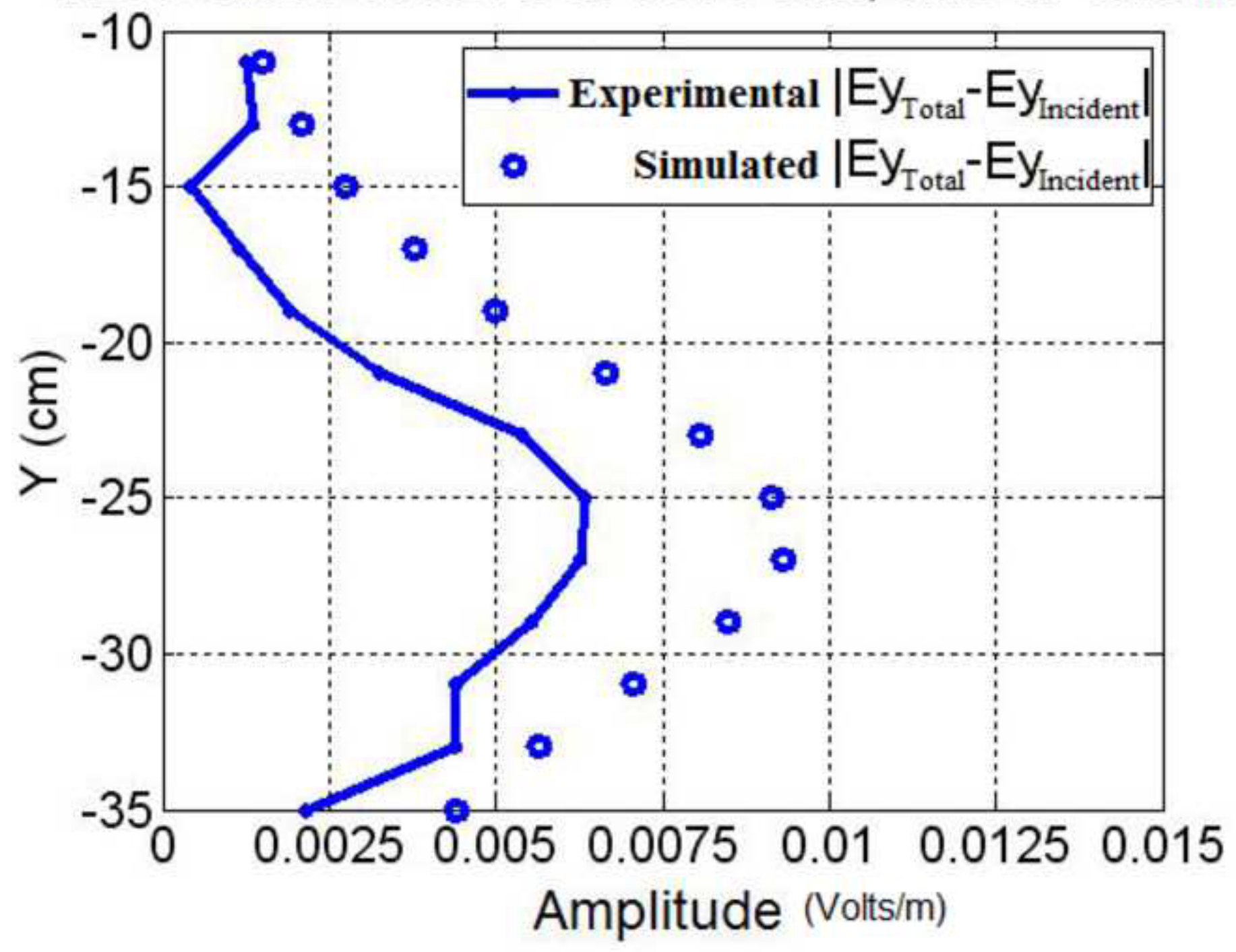




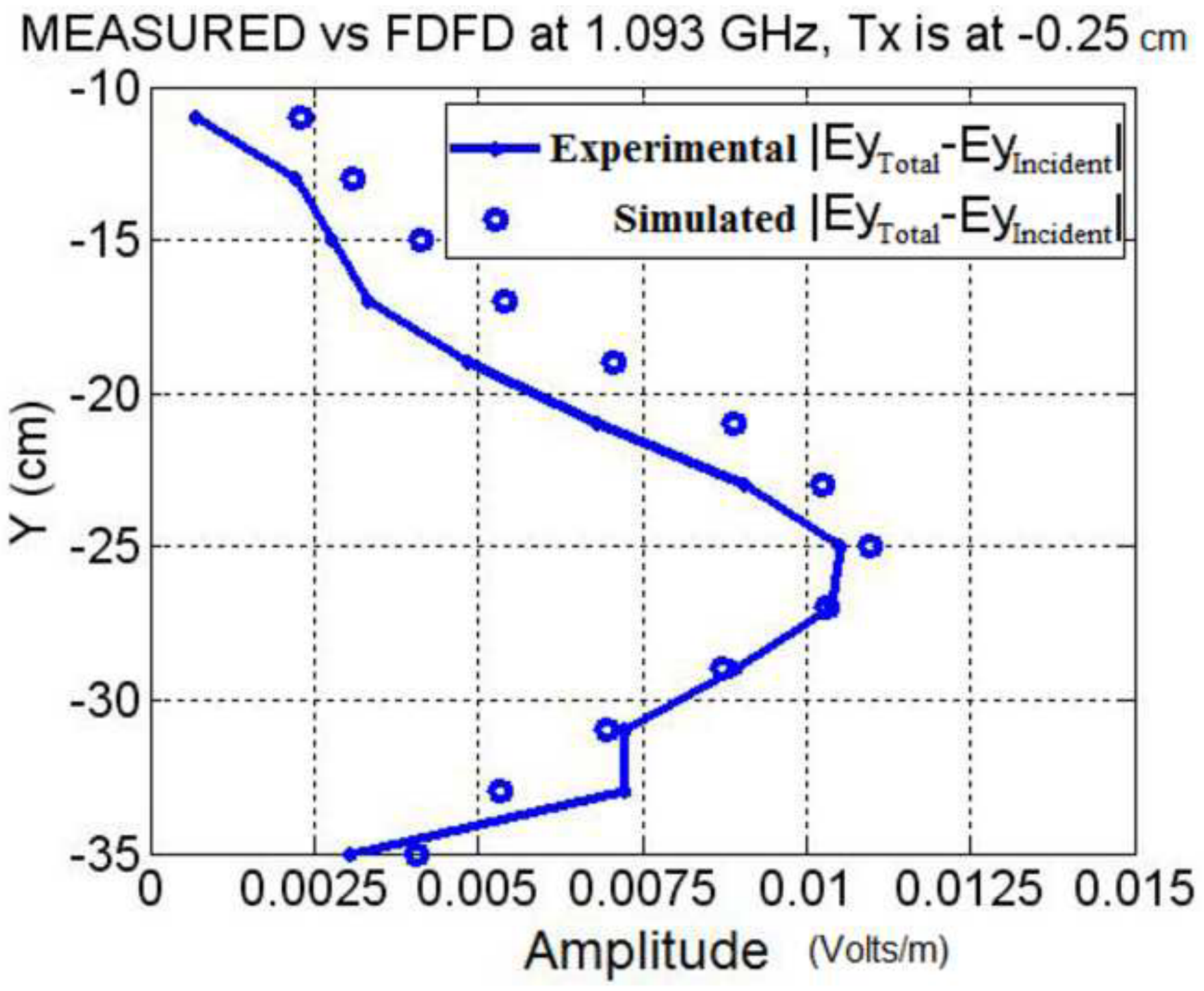




\section{MEASURED vs FDFD at $1.093 \mathrm{GHz}$, Tx is at $-0.31 \mathrm{~cm}$}

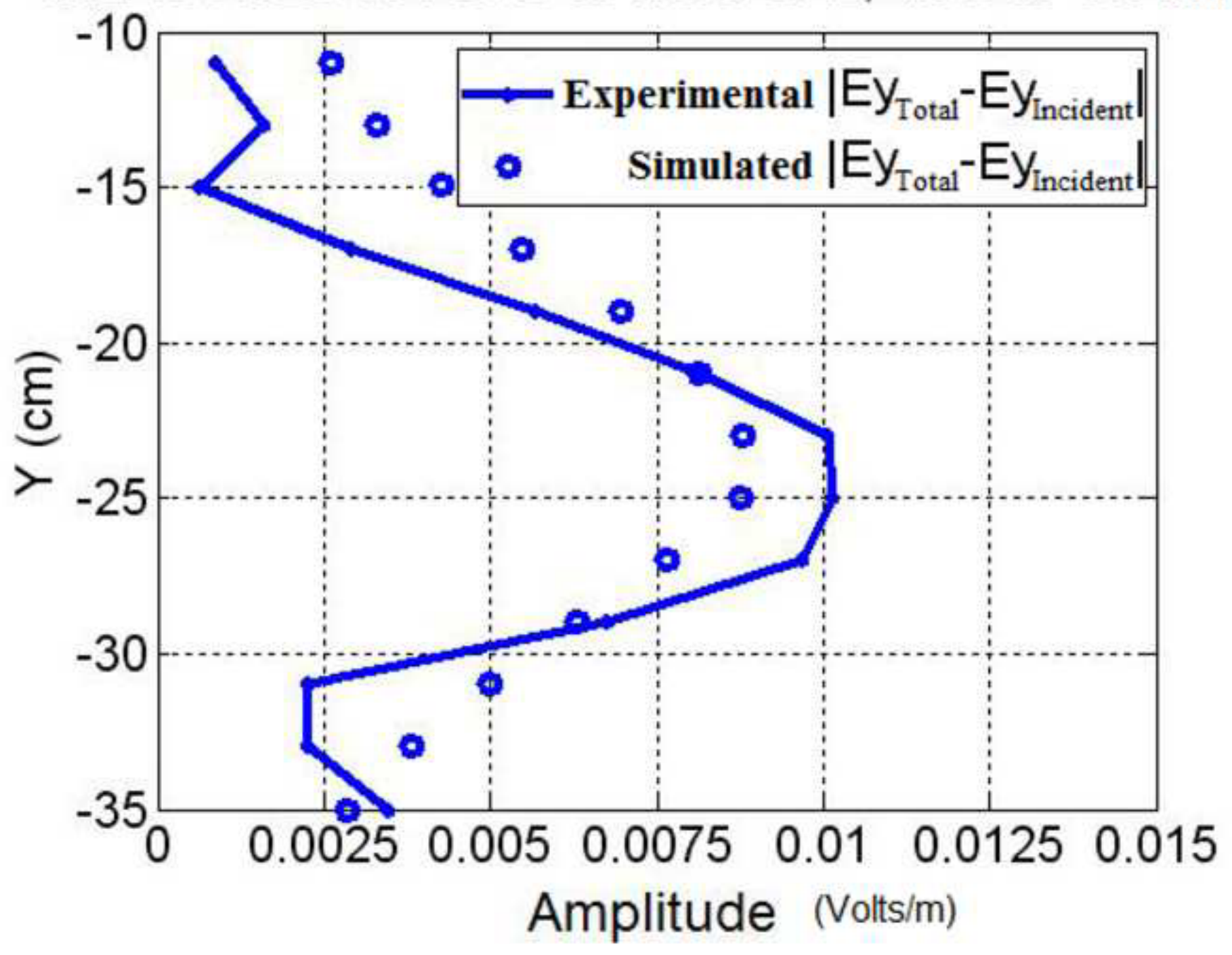

